\title{
Vejez, ¿un final "triste" para una historia de vida?. Revisión de estudios sobre la depresión en los adultos mayores
}

\author{
Lizeth Cristina Martínez Baquero ${ }^{1}$, Claudia Patricia Moreno Heredia ${ }^{2}$ \& Xiomara \\ Esperanza Sánchez Velásquez ${ }^{3}$
}

\begin{abstract}
Since the end of the last century the world is faced with the phenomenon of increased life expectancy and therefore of the aging population. The objective of the present article is to present a review of empirical studies on the approach to the depression during adulthood increased, issue which has been left behind due to the set of myths, stereotypes and prejudices that surround it. Contemplated a considerable number of articles, whose results are related to the vulnerability of older adults suffer from depression, due to the various changes they are experiencing. Some of the factors that influence the occurrence of depressive symptoms are: problems of health, retirement, consumption of alcohol, difficulties in interpersonal relations, family dysfunction, sensory impairments, cognitive impairment, absence of low-income housing and economic as well as being linked to homes of step. In relation with the sex the studies show than the affective disorders in the woman are most frequently than the mens.
\end{abstract}

Editado por| William Alejandro

Jiménez-Jiménez,

Universidad de Boyacá, Colombia.

Revisado por|Ana María Ávila-

Matamoros,

Fundación Universitaria Konrad

Lorenz, Colombia.

Keywords | Adulthood greater, depression, risk factors, old age, suicide.

\section{${ }^{1}$ Lizeth Cristina Martínez Baquero $[\bowtie]$}

${ }^{2}$ Claudia Patricia Moreno Heredia

${ }^{3}$ Xiomara Esperanza Sánchez Velásquez
Psicóloga Universidad Pedagógica y Tecnológica de Colombia. Master en Psicología, Clínica A.E.P.C. Directora de Investigaciones Facultad de Ciencias Humanas y Educativas. Email: Icmartinez@uniboyaca.edu.co Psicóloga Universidad de Boyacá.

Psicóloga Universidad de Boyacá, especialista en Psicología Jurídica

E $\| 72$ 


\section{Introducción}

Desde finales del siglo pasado, la humanidad se enfrenta con un fenómeno de envejecimiento poblacional. La vejez es una etapa importante, final en la vida del ser humano, como preocupación esencial reclama la atención de la sociedad (Villanueva, 2005).

Pareciera ser que a medida que las personas envejecen, la sociedad las olvida, los sujetos mayores pierden vigencia en el mundo donde están inmersos convirtiéndose en una "carga" para sus familiares e incluso para la sociedad, lo cual, evidentemente constituye un factor de riesgo importante en la salud mental de los mayores. Olvidando que son sujetos activos y no únicamente actores pasivos de la marcha del tiempo. De hecho, la mayoría de investigaciones con adultos mayores centran su interés en la Depresión, olvidando otros muchos tópicos que pueden ser interesantes de abordar.

En este artículo se pretende hacer una aproximación al tema desde una revisión a los estudios empíricos sobre la depresión en los adultos mayores realizados desde el año 2004 hasta el 2013, la búsqueda de artículos se realizó en aquellas publicaciones electrónicas de revistas indexadas, el abordaje del tema inicia por identificar la prevalencia, luego se abordan los factores de riesgo reconocidos para la depresión, continuando con las características del trastorno en esta edad, para terminar con los estudios que relacionan la depresión con los trastornos cognitivos.

\section{Prevalencia de la Depresión en la Adultez Mayor}

Es común relacionar ancianidad y depresión dadas las características que la edad implica ya que en muchos aspectos, constituye "pérdida". Pareciera que el anciano pierde juventud, pierde capacidad, pierde seres queridos, pierde, pierde. Los estudios revisados indican cómo están fuertemente relacionadas la vejez y la depresión. Los párrafos que siguen mostrarán los resultados más relevantes respecto a la prevalencia de la depresión en este grupo poblacional.

Sin embargo, la depresión no es una problemática exclusiva de las personas mayores. De hecho, la Organización Mundial de la Salud - (OMS) - (2012) estimó que la depresión es una enfermedad cotidiana que afecta a un promedio de 350 millones de sujetos en el mundo de diferentes rangos de edad, se presenta como una problemática de salud delicada, y que su padecimiento 
influye en las actividades diarias de los sujetos. Por ende puede conducir al suicidio, siendo éste una de las principales causas de muerte de quienes padecen depresión.

Según publica la OMS en su centro de prensa virtual (2014), la proporción mundial de adultos mayores aumenta rápidamente en todo el mundo. Así, sus cálculos advierten que entre el año 2000 y 2050 dicha proporción pasará de 11 a 22\%. El aumento previsto es de 605 millones a 2000 millones de personas mayores de 60 años con depresión.

Guerra, Ferri, Sosa, Salas, Gaona, González, Rojas de la Torre y Prince (2009) en su estudio realizado comparan la prevalencia de depresión entre poblaciones de adultos mayores de México, Perú y Venezuela. Sus resultados muestran un nivel considerable de depresión grave, correlacionado con enfermedades físicas e historias previas de depresión. Las correlaciones no son datos muy sorprendentes, lo que llama la atención de su estudio es identificar que la mayoría de los adultos deprimidos, lo estuvieron durante buena parte de su vida y nunca recibieron la atención requerida.

Es así como Sánchez y Castañeda (2008), estimaron la prevalencia de los trastornos del ánimo en atención primaria, (utilizando la escala Yesavage) y la asociación de los trastornos del ánimo a otras variables, para buscar el perfil del anciano. Encontrando que la prevalencia de depresión fue de un $16 \%$, de los sujetos participantes de su estudio. Así mismo, se evidencia que el anciano al cual se debe prestar mayor atención es el que cumpla el siguiente perfil: sexo femenino, edad 75 años, estudios primarios o inferiores, dependencia para alguna de las actividades básicas de la vida diaria, antecedentes personales previos de depresión, tratamiento actual con antidepresivos, toma de fármacos diariamente y aislamiento social.

Para Pérez y Arcia (2007) la depresión predomina en mayor nivel en ancianos de 60 a 69 años, del sexo femenino, viudos, jubilados y con un bajo nivel de escolaridad. Así mismo encontraron que en un $82,6 \%$ la depresión se presenta simultáneamente con otras enfermedades, como las psicosomáticas y los trastornos de ansiedad. Para ellos la jubilación y la edad son los factores que más se relacionan con la depresión en los adultos mayores.

En Estados Unidos, estudios epidemiológicos evidencian que la prevalencia de la depresión es mayor en pacientes institucionalizados ya sea en hospitales u hogares. Según Dechent (2006) en-

$\boldsymbol{E} \mid 74$ 
tre 1 y $4 \%$ de la población anciana no hospitalizada presenta depresión. Se estima que el 0-15\% por año, mientras que en pacientes hospitalizados el porcentaje aumenta al $10-12 \%$ y $12-14 \%$.

En la investigación de Gómez, Bohórquez, Pinto, Gil, Rondón y Díaz (2004), se analizó la prevalencia del síndrome depresivo y los factores asociados con la depresión en Colombia entre los años 2000 y 2001. La muestra estuvo conformada por 116 adultos mayores. La depresión se clasificó en breve, subclínica o clínica (leve, moderada o grave). El instrumento aplicado fue una encuesta en la cual se evaluaron más de 500 variables: identificación, revisión general y demográfica, percepción del estado de salud, estado de salud, salud mental y consumo de sustancias adictivas, capacidad de respuesta del sistema de salud y pruebas de calibración. Para el tamizaje de la depresión se aplicaron los criterios de la 10a edición del CIE-10 y del DSM-IV.

Los resultados arrojaron que el $10 \%$ de las personas estudiadas presentaron algún episodio depresivo en los 12 meses previos a la encuesta y 8,5\% sufrieron alguno durante el último mes. Hubo una mayor proporción de mujeres con depresión en ambos períodos y las mayores prevalencias se encontraron en las personas mayores de 65 años. Con esta investigación se pone de manifiesto cómo la depresión es una afección frecuente en Colombia y que se deben poner en marcha medidas dirigidas a reducir el riesgo de depresión, especialmente en mujeres y en personas mayores de 65 años de edad.

En este sentido, es conveniente entender como lo señala Del Barrio et.al (2004), que en los últimos tiempos se ha confirmado la incidencia de los trastornos del estado de ánimo no solo en la edad adulta sino también en la infancia y juventud. Además se ha comprobado que más de la mitad de los casos de adultos con depresión tienen antecedentes infantiles de dicha sintomatología.

Los estudios anteriormente analizados muestran que el sexo femenino presenta el doble de posibilidades de desarrollar un trastorno depresivo en comparación con el sexo masculino. Igualmente los resultados sugieren como a mayor edad, mayor probabilidad de presentar depresión. Los estudios exponen además que la dependencia, el vivir solos, y la baja escolaridad son en su orden variables altamente relacionadas con la depresión, que siguen al sexo, edad y jubilación. Las investigaciones anteriormente citadas revelan el suicidio altamente relacionado con la depresión y la vejez, pero también muy subvalorado en la investigación científica. 


\section{Factores de Riesgo}

En este aspecto Fernández y Méndez (2007) identificaron los estilos de vida y factores de riesgo para la salud de las personas adultas mayores del proyecto creles - Costa Rica 2004-2006 y los resultados obtenidos por ellos describen características de riesgo diferenciadas por sexo, donde las mujeres presentan mayor riesgo a la depresión, deterioro cognitivo, mayor número de limitaciones físicas, estar sin pareja, y en general una auto percepción de su salud como de regular a mala.

De igual manera en la Investigación de Patiño y Cardona (2007), se encuentra una asociación estadísticamente significativa, entre la depresión y la percepción de la situación económica como mala. Se encontró que con mayor frecuencia la depresión se da en el sexo femenino.

En este mismo sentido García, Guridi, Medina y Dorta (2007), reportaron en su caracterización de las variables psicosociales asociadas a la depresión en ancianos y describieron el tipo de depresión presente en el grupo de estudio, utilizando entrevistas al médico, a la familia y a la enfermera. Los investigadores recogen un número considerable de variables socio demográficas dentro de las que se incluyen: edad, sexo, escolaridad, ocupación, economía y otras variables como: convivencia, maltrato, función que ocupa en la familia, estado emocional, actividades de la vida diaria, limitaciones físicas, principales síntomas psicosomáticos y utilización del tiempo libre.

En esta investigación se identificaron que tanto características personales como contextuales pueden convertirse en factores de riesgo para la depresión. Así mismo que la jubilación ocupa un lugar muy importante en el desarrollo del trastorno depresivo.

En este sentido investigaciones como la de Castro, Ramírez, Aguilar y Díaz (2006) quienes escogieron una muestra no probabilística de 511 sujetos de 60 años de edad y más, obtuvieron como resultado la prevalencia de depresión en un $41.7 \%$. Observaron un riesgo fuerte para desarrollar depresión en los individuos expuestos a bajo apoyo social, económicamente improductivos y la no pertenencia a programas gubernamentales de asistencia social.

En la Investigación de Molina (2005) se encontró que de 100 adultos mayores Guatemaltecos, el $70 \%$ de la muestra, indicó una presencia mínima de síntomas depresivos, el 25\% reflejó una 
depresión media y el 5 \% presentó una depresión moderada. No se encontraron diferencias significativas entre hombres y mujeres ni entre estado civil, pero sí en la variable de con quien vive ya que las personas que viven con un pariente (hijos, sobrinos, nietos) presentan un nivel más bajo de depresión en comparación con las personas que viven solas.

Para Tuesca, Fierro, Molinares, Oviedo, Polo y Sierra (2004), los adultos mayores son una población vulnerable a sufrir de depresión debido a todos los cambios que las personas atraviesan. Algunos de los factores que influyen en la aparición de síntomas depresivos son: problemas de salud, jubilación, consumo de alcohol, dificultades en las relaciones interpersonales, disfunción familiar, limitaciones sensoriales, ausencia de vivienda y bajos ingresos económicos.

Para Cassano (2002, citado por Doglas, sf) La depresión a menudo coexiste con otras condiciones médicas como enfermedad cardiaca, derrame cerebral, cáncer, VIH/SIDA, diabetes y la enfermedad de Parkinson. Así mismo, distintos estudios han demostrado que quienes padecen depresión, junto con otras enfermedades médicas graves, tienden a presentar síntomas más graves, tanto de la depresión como de las enfermedades.

Por lo anterior se observa entonces que las variables con las que está asociada la depresión, así como los factores de riesgo en los adultos mayores se relacionan con: el proceso de jubilación, la percepción de una situación económica precaria y la percepción de "pérdida" de la juventud, visibilizada en los diferentes cambios físicos. Así mismo se identifica que son las mujeres quienes más presentan el trastorno y que dentro de los síntomas característicos de la depresión, la tristeza es el que mayormente se presenta en el grupo de adultos mayores deprimidos.

\section{Características de la depresión en la Vejez}

Aunque los diferentes trastornos del estado del ánimo tienen síntomas similares, también se configuran características particulares, de acuerdo con la edad. En la vejez la depresión muestra algunas diferencias en comparación con la adultez.

En este sentido la investigación de Álvarez (2010) analizó la depresión en un grupo de ancianos institucionalizados del Hospital Salvador Allende, identificando las principales características del trastorno. La muestra estuvo conformada por 64 adultos mayores de 60 años que tenían como diagnóstico: trastorno bipolar en fase depresiva, trastorno depresivo (leve, moderado, 
profundo), trastorno depresivo recurrente, trastorno del estado de ánimo debido a enfermedades médicas y trastorno de adaptación depresivo.

Se encontró nuevamente que en cuanto al sexo las mujeres parecen más vulnerables a la depresión que los hombres, situación que está relacionada con el rol social que han venido desempeñando las mujeres a lo largo de la historia. La soledad fue un factor de riesgo importante considerándose uno de los más peligrosos para la conducta suicida. Los principales factores de riesgo identificados en esta investigación fueron: ancianos que viven solos, la existencia de enfermedad orgánica asociada y la jubilación.

En el estudio de Peña, Herazo, y Calvo (2009) se revisaron los aspectos clínicos más relevantes del trastorno depresivo en los ancianos con el fin de facilitar al médico de atención primaria las herramientas básicas para un adecuado diagnóstico y tratamiento, y de esta manera ayudar a reducir el impacto negativo de este trastorno en la comunidad. Los resultados de esta revisión evidenciaron que la presencia de un trastorno depresivo en personas mayores de 65 años aumenta la mortalidad en este grupo etareo y produce un impacto negativo en la calidad de vida, constituyéndose como un problema de magnitud mundial.

Por su parte Alcalá, Camacho y Giner (2007) analizaron las posibles diferencias en la afectividad de la depresión de la tercera edad respecto de la depresión de la edad adulta. Encontrando que el grupo depresivo de ancianos puntuó más en afectividad negativa, miedo, hostilidad, culpa, tristeza y fatiga, mientras que el grupo de control obtuvo valores superiores en las sub-escalas de afectividad positiva, jovialidad, autoconfianza y serenidad.

Es importante mencionar la investigación realizada por Ávila, Melano, Pallete y Amieva (2007) en la que se buscó determinar la relación entre síntomas depresivos y dependencia funcional. En 880 individuos, encontrando que los ancianos más dependientes para sus actividades son los que están mayormente afectados por síntomas depresivos.

Por otro lado, Márquez, Soriano, García y Falcón (2005) identificaron la frecuencia y factores de riesgo asociados a la depresión en los adultos mayores en una unidad de primer nivel en la zona urbana de la Ciudad de México. La muestra estuvo compuesta por 384 adultos mayores de 60 años. Las variables identificadas fueron: sexo, edad, situación laboral, dependencia económica,

$\boldsymbol{E} \| 78$ 
función familiar, pluri-patología y actividad física. Se evalúo el estado depresivo con la escala geriátrica de Yasavage y Brink.

Los principales resultados de esta investigación fueron que la frecuencia de depresión se dio en un $72,1 \%$ de los sujetos participantes, se corrobora la mayor frecuencia en mujeres, jubilados, pensionistas, sedentarios, con familias disfuncionales y con dependencia económica.

Los estudios presentados en el apartado que termina, muestran la relación importante existente entre la depresión y el riesgo de suicidio en los adultos mayores, relacionándolo con la soledad. Se encuentra cómo los ancianos más olvidados por sus familias, el Estado y la sociedad son los más vulnerables a acabar con sus propias vidas. De igual manera las investigaciones presentan cómo en los adultos mayores la sintomatología de afecto negativo: tristeza, miedo e ira son más frecuentes en comparación con otras características de la depresión. Así mismo, los Adultos mayores deprimidos, en su gran mayoría tienen otra enfermedad crónica y son dependientes en actividades básicas cotidianas.

\section{Vejez, Depresión, Suicidio}

La Organización Mundial de la Salud afirma que existe aumento de la prevalencia de intento suicida con la edad, la tasa aumenta en mayores de 75 años. En este grupo poblacional los suicidios representan una alarma en cuanto a los problemas psicológicos, físicos y sociales.

Un estudio clásico en el tema de Depresión Vejez y suicidio lo constituye el realizado por Wagner, Gallo y Delva, (1999) realizado en México, en el que se observa que la prevalencia de suicidio es más alta entre hombres de 75 años de edad o más, y entre jóvenes de entre 15 y 19 años. La prevalencia de la depresión mayor es más alta en hombres que en mujeres (4.4/0.8). Lo que resulta más problemático es que son muy pocas las personas con depresión que recibieron o reciben tratamiento.

En la investigación de Vega, Pereda, Vega y Alonso (2003) se analizó la tendencia y factores de riesgo del suicidio en el adulto mayor en una muestra de 50 fallecidos por suicidio, mayores de 60 años pertenecientes al Policlínico Docente "José Ávila Serrano" de Velasco, durante el periodo 1987- 2000. Los resultados de este estudio indicaron que factores de riesgo como: vivir solo, sin 
pareja estable y la procedencia rural son los principales factores asociados al suicidio. Además como principal conclusión afirmaron que las tasas de suicidio en los ancianos son mayores que en cualquier otro grupo de edades, pues llegan quizás a duplicar la de la población en general.

\section{Deterioro Cognitivo y Depresión}

Esta es otra variable que se ha identificado como altamente relacionada con la problemática de la vejez.

La investigación realizada por Córdoba (2008), tuvo como objetivo identificar la relación existente entre el estado de los procesos cognitivos y el nivel de depresión de los adultos mayores. La muestra estuvo conformada por 67 adultos mayores pertenecientes a los diversos pabellones de la Institución. Se utilizaron 2 instrumentos para la evaluación de la depresión: la Escala de Depresión Geriátrica de Beck, y para evaluar el nivel cognitivo y la prueba de MinimentalMini examen cognoscitivo de Geneco que evalúa las capacidades cognitivas teniendo en cuenta aspectos tales como orientación, retención, atención, cálculo, evocación, lenguaje y praxias.

Dentro de los resultados se encontró que los sujetos con mayores índices de deterioro cognitivo, tienden a padecer mayores síntomas depresivos, en comparación con sujetos con menor deterioro. Así mismo se encontró que el deterioro cognitivo aumenta con la edad y que se muestra como una característica propia de la vejez.

Estos resultados se relacionan con lo encontrado en la investigación realizada por Marcia Vásquez Gutiérrez (2007) que tuvo como intención abordar el infra diagnóstico de demencia y el escaso uso de instrumentos que evalúan la esfera cognitiva con el objetivo de caracterizar pacientes con deterioro cognitivo según edad, sexo, escolaridad, factores de riesgo asociados, esfera cognitiva afectada y severidad de la misma. La muestra estuvo constituida por un total de 743 adultos mayores pacientes, 129 de ellos con deterioro cognitivo. La mayoría estuvo entre 75 a 79 años. Se encontró que entre el $4 \%$ y el 5\% de las personas mayores de 65 años padecen de demencia con una marcada incapacidad.

Otra Investigación encontrada es la de Hervás y García (2005), con el objetivo de conocer la influencia del estado cognitivo en la presencia de diferentes factores de fragilidad en el anciano. La muestra estuvo constituida por un total de 147 ancianos mayores de 65 años. Como principal

\section{E $\| 80$}


conclusión de esta investigación se obtuvo que el deterioro cognitivo marca la aparición de forma significativa de factores de fragilidad en el anciano como las caídas, incontinencia urinaria, hospitalización-reingresos hospitalarios y depresión.

\section{Vejez, Factores protectores y Prevención Temprana}

Si bien la revisión realizada hasta el momento, enfatiza como es su objetivo en presentar estudios relacionados con la depresión en este grupo etario, la exploración que sigue permitirá brindarle al lector una reflexión sobre la importancia de la intervención temprana de los trastornos emocionales, para procurar en los adultos mayores un "final" distinto a sus historias de vida.

En la búsqueda realizada, se encontraron un buen número de investigaciones que caracterizan los factores de riesgo de la depresión y un menor número de investigaciones sobre los factores protectores de la depresión, como elementos que enmarcan una perspectiva positiva del envejecer.

En este sentido la Asociación Norteamericana de Psiquiatría Geriátrica (citada por Herrera et.al 2010). Argumenta que las personas deben prepararse continuamente para los cambios en cualquier ciclo vital y especialmente para los cambios que trae consigo la vejez como la jubilación, cambios de vivienda, muertes del cónyuge, amigos, etc, por lo que las actividades psicoeducativas por parte de familiares y personal asistencial serán muy útiles.

De esa manera se deben mantener y promover en el anciano las relaciones con los amigos y la inclusión en actividades de agrado, lo cual permitirá la actividad mental y física del anciano. Sin duda el apoyo familiar que se le provea al adulto mayor permitirá que en momentos de soledad se le contenga y permita la expresión emocional.

En este sentido para Herrera et.al (2010) La Educación Física y los deportes ofrecen grandes posibilidades para los adultos mayores, así como cualquier otra forma de participación que incluya el ejercicio físico se consideran una forma adecuada de prevenir cualquier enfermedad física o mental entre ellas la depresión.

Para Sánchez y Castañeda (2008) la prevención de la depresión en los adultos requiere, una sensibilización a la sociedad y al personal asistencial sobre la vejez, así como un trabajo multisectorial y multidisciplinar para la atención del adulto mayor. Además de la necesidad de 
observar el envejecimiento desde una perspectiva de educación para la salud, lo cual permitirá la articulación de niveles y actores de toda la sociedad que rodea a los adultos mayores.

Con relación a este aspecto, se encontró el estudio realizado por Tuesca, Fierro, Molinares, Oviedo, Polo y Sierra (2004), este estudio tuvo como objetivo determinar el papel que juegan los grupos de socialización como factor protector para la depresión en personas ancianas. En él participaron 602 personas ancianas de entre 60 y 94 años. Los resultados indicaron que aquellos sujetos que gozaban de la participación en actividades de socialización, tenían menor riesgo de padecer depresión. En cuanto a las limitaciones sensoriales como ceguera y sordera, la soledad, ausencia de vivienda y bajos ingresos económicos, en su orden se comportaron como factores de riesgo para padecer depresión, lo cual muestra la necesidad de involucrar a los ancianos en actividades socializadoras que les permitan sentirse útiles y no excluidos de los diferentes grupos sociales.

\section{Conclusiones}

Para finalizar la presente revisión se presentan algunas conclusiones generales de las investigaciones y estudios reseñados:

La depresión no es una problemática exclusiva de la adultez mayor, sin embargo los estudios revisados muestran que entre el 8 y $20 \%$ de los adultos mayores de 60 años presentan algún tipo de sintomatología depresiva, en comparación con los niños en quienes dicha prevalencia es aproximadamente entre el 10 y $20 \%$ (Vinaccia et al.2006), en los jóvenes aumenta, especialmente en edades comprendidas entre los 14 y 16 años hasta un 25\% (Pérez, Ibáñez, Reyes, Atuesta, Suárez 2008; Pandolfoa, Vázqueza, Mása, Vomeroa, Aguilara y Belloa, 2011) lo cual llama la atención no por las cifras si no porque lleva a preguntas interesantes para las ciencias del cuidado de la salud mental referentes a la efectividad de los programas interventivos para la depresión en los diferentes grupos de edad, el curso de la depresión y los factores contextuales que enmarcan la predisposición y riesgo de padecer depresión.

Es evidente a través de las diferentes investigaciones, como "ser mujer" y el aumento de la edad, son factores de riesgo para padecer depresión, si a esto se le suma, ser jubilado, estar abandonado por la familia o haberla perdido y adicionalmente padecer de deterioro cognitivo,

\section{E 82}


se tiene la suma "perfecta" para padecer de alguna forma de trastornos del estado de ánimo durante la última etapa de la vida.

Se determinó también, que el medio ambiente influye de forma importante en el control y prevención de los estados depresivos del anciano y a su vez que la integración familiar y social de las personas de edad avanzada, protegen en buena medida al adulto mayor de padecer depresión.

Las investigaciones reseñadas ponen de manifiesto la necesidad de que el adulto mayor ponga en práctica un mejor control emocional y afectivo que prevenga el padecimiento de los trastornos afectivos. Incluso es necesario implementar nuevas medidas de atención personal y comunitaria, encaminadas al diagnóstico y tratamiento precoces de los estados depresivos de los adultos mayores.

Preocupa enormemente que el sentirse excluido y no activo dentro de la sociedad genera en las personas y mas aún en los ancianos sentimientos de tristeza, ira y desolación, siendo éstos los más característicos en la depresión del adulto mayor en comparación con otros síntomas padecidos por adultos en trastornos depresivos.

Además, existe una asociación fuerte entre jubilación y estado afectivo de insatisfacción, por las pérdidas de rol, estatus social y familiar, así como la disminución del poder adquisitivo y acumulamiento de necesidades materiales no resueltas. Así mismo, alrededor de los 65 años se plantea que el $50 \%$ de los individuos presentan alguna alteración psíquica en la que predominan la ansiedad y la depresión. Esto refleja que existe un mayor nivel de vulnerabilidad en el adulto mayor frente a situaciones comunes que se pueden presentar durante esta etapa.

Los investigadores concluyeron que el estudio de la vejez dentro de la comunidad psicológica es fruto de una clara evidencia social: la población del mundo occidental a partir del siglo XX ha mejorado en su esperanza de vida. Lo cual amerita que el mundo se interese por proteger la salud y el bienestar de la población adulta.

Así como que el envejecimiento mundial de la población y los fenómenos naturales que la acompañan hacen de los ancianos un grupo de alto riesgo para padecer depresión. Los resultados muestran la necesidad de emprender acciones preventivas desde la infancia y la adolescencia 
para disminuir la prevalencia durante la vejez. Así mismo la imperante necesidad de mitigar desde las políticas públicas los factores de riesgo susceptibles de ser modificados durante cualquier etapa de la vida.

Se hace necesario continuar proponiendo planes de intervención que involucren la estimulación cognoscitiva, la inclusión en actividades físicas y pasatiempos con los ancianos y sus familias que permitan brindarles una mejor perspectiva de vida para sus últimos días.

Porque finalmente todos vamos a ser ancianos.

\section{Referencias}

Alcalá, V., Camacho, M \& Giner, J. (2007). Afectos y depresión en la tercera edad. Psicothema, 19(1), 49-56. Recuperado de http://redalyc.uaemex.mx/pdf/727/72719108.pdf

Álvarez, Y. (2010). Depresión, fenómeno oculto y latente. Análisis de la depresión en el anciano. Revista del Hospital Psiquiátrico de La Habana, 7 (3). Recuperado de http://www.revistahph.sld.cu/hph0310/hph08310.html

Arango, J. \& Fernández, S. (2003). Depresión en la enfermedad de Alzheimer. Revista latinoamericana de psicología, 35(1), 41-54. Recuperado de http://redalyc.uaemex.mx/pdf/805/80535104.pdf.

Ávila, J., Melano, E., Pallete, H \& Amieva, H. (2007). Síntomas depresivos como factor de riesgo de dependencia en adultos mayores. Salud pública de México, 49 (5), 367-375. Recuperado de http://www.medigraphic. com/pdfs/salpubmex/sal-2007/sal075g.pdf

Campos, J., Ardanaz, J \& Navarro, A. (2004). Depresión en pacientes de edad avanzada. Dos ámbitos: un centro sociosanitario y un programa de soporte domiciliario. Revista Española de Geriatría y Gerontología, 39 (4), 232-239. Recuperado de http://www.elsevier.es/sites/default/files/elsevier/pdf/124/124v39n 04a13064974pdf001.pdf.

Canto, H. \& Castro, E. (2004). Depresión, autoestima y ansiedad en la tercera edad: un estudio comparativo. Enseñanza e Investigación en Psicología, 9(2), 257-270. Recuperado de http://www.redalyc.org/articulo. oa?id $=29290204$

E $\| 84$ 
Castaño, L.; Cardona, J. \& Torres, L. (2011). Eficacia de un programa terapéutico de expresión plástica en la depresión de los adultos mayores del Centro de Bienestar de Ancianos San José Pereira. (Tesis de pregrado - Universidad tecnológica de Pereira). Recuperado de http://repositorio.utp.edu.co/tesisdigitales/ texto/7901926C346e.pdf

Castro, M.; Ramírez, S.; Aguilar, L. \& Díaz, V. (2006). Factores de riesgo asociados a la depresión del adulto mayor. Revista de Neurología, Neurocirugía y Psiquiatría, 39(4). Recuperado de http://www.imbiomed.com. $\mathrm{mx} / 1 / 1 /$ articulos.php?method=showDetail\&id_articulo=41190\&id_seccion

Contreras, D.; Moreno, M.; Martínez, N.; Araya, P.; Livacic, R. \& Vera, V. (2006). Efecto de una intervención cognitivo-conductual sobre variables emocionales en adultos mayores. Revista latinoamericana de psicología, 38(1). Recuperado de http://redalyc.uaemex.mx/src/inicio/ArtPdfRed.jsp?iCve=80538103

Córdoba, A. (2008) Relación entre los procesos cognitivos y el nivel de depresión en las adultas mayores institucionalizadas en el asilo San Antonio de Bucaramanga. Universitas Psichologica, 7(1), 271-281. Recuperado de http://sparta.javeriana.edu.co/psicologia/publicaciones/actualizarrevista/archivos/19v7n1_cerqueracordoba.pdf.

Del Barrio, V. (2004). La depresión infantil: factores de riesgo y posibles soluciones. Editorial Aljibe.

Dechent, C, (2006). Bases de la medicina Clínica. Unidad Geriátrica. Recuperado de http://www.basesmedicina.cl/ geriatría/06_trast_animo/geria_trast_animo.pdf

Díaz, M \& Peralta, H. (2008). Detección precoz del deterioro cognitivo ligero de la tercera edad. Psicothema, 20 (3), 438-444. Recuperado de http://www.psicothema.com/pdf/3505.pdf

Fernández, X. \& Méndez, E. (2007). Estilos de vida y factores de riesgo para la salud de las personas adultas mayores del proyecto creles- costa rica 2004-2006. Población y salud en Mesoamérica, 5 (número especial), 1-17. Recuperado de http://www.redalyc.org/pdf/446/44659906.pdf

García, F.; Guridi, M.; Medina, A. \& Dorta, Z. (2007). Comportamiento de la depresión en el adulto mayor. Revista de Psicología científica. Recuperado de http://www.psicologiacientifica.com/bv/psicologiapdf301-comportamiento-de-la depresion-en-el-adulto-mayor.pdf 
Gómez, C., Bohórquez, A., Pinto, D., Gil, J., Rondón, M \& Díaz, N. (2004) Prevalencia de depresión y factores asociados con ella en la población colombiana. Revista Panamericana de Salud Pública, 16(6), 378-386. Recuperado de http://www.scielosp.org/pdf/rpsp/v16n6/23682.pdf

Guerra, M., Ferri, C., Sosa, A., Salas, A. Gaona, C., González, V., Rojas de la Torre, G. y Prince, M. (2009). Late-life depression in Peru, Mexico and Venezuela: the 10/66 population-based study. The British Journal of Psychiatry, 195(6), 510-515. Recuperado de http://www.ncbi.nlm.nih.gov/pubmed/19949200

Herrera, L., Prieto, A. \& Guerra, T. (2010). Plan de Intervención Comunitario dirigido al adulto mayor en el Consejo Popular Rafaelito del municipio, Cumanayagua. Recuperado de http://www.efdeportes.com/efd147/ plan-de-intervencion-comunitario-dirigido-al-adulto-mayor.htm

Hervás, A. \& García, E. (2005). Situación cognitiva como condicionante de fragilidad en el anciano: perspectiva desde un centro de salud. Anales del Sistema Sanitario de Navarra, 28(1), 35-47. Recuperado de http://scielo.isciii.es/scielo.php?pid=S1137-66272005000100004\&script=sci_arttext

López, J., Galdón, M., Fernández, C., Escobar, F., Montoya, J., Boix, C., Montes, M., Blas, L., Cremades, M., Campo, J. \& Arnalich, F. (2005). Diseño y validación de un cuestionario para la detección de depresión mayor en pacientes ancianos. Gaceta Sanitaria, 19(2), 1103-112. Recuperado de http://www.scielosp.org/ pdf/gs/v19n2/original2.pdf

Marcia, A., Vásquez, E. \& Gutiérrez, M. (2007). Deterioro cognitivo en el adulto mayor. Instituto de información científica y tecnológica. Revista ciencias Holguín, 13(4). Recuperado de http://www.redalyc.org/src/ inicio/ArtPdfRed.jsp?iCve=181517998004

Márquez, S.; Soriano, A.; García, M. \& Falcón, M. (2005). Depresión en el adulto mayor: frecuencia y factores asociados. Revista de atención primaria de salud de México, 36(6). Recuperado de http://www.elsevier. es/es/revistas/atencion-primaria-27/depresion-adulto-mayor-frecuencia-factores-riesgo-asociados13079874-cartas-investigacion-2005

Martínez, O., Rodríguez, X., Gillot, A., \& Rodríguez, L. (2007). Comportamiento de la depresión en el adulto mayor del policlínico enrique Betancourt. Revista avanzada científica, 10. Recuperado de http://avanzada. idict.cu/index.php/avanzada/article/view/168/175

\section{E 86}


Molina, I. (2005). Depresión en el adulto mayor en miembros del centro de atención médica integral para pensionados (CAMPI) en Guatemala. Universidad Francisco Marroquín, Guatemala. Recuperado de http:// portal.salud.gob.mx/descargas/pdf/pns_version_completa.pdf

Morales, J.; Rodríguez, R.; Ortega L. \& Lazcano, G. (2006). Hacia la prevención de la depresión. A partir del estudio de los factores asociados a la presencia del síntoma depresión. Revista de Especialidades Médico-Quirúrgicas, 11(2). Recuperado de http://redalyc.uaemex.mx/src/inicio/ArtPdfRed.jsp?iCve=47311956009

Muñoz, L., Price, Y., López, M., Ramírez, M. \& Costa M. (2010). Vivencia de los cuidadores familiares de adultos mayores que sufren de depresión. Revista Escolar de Enfermedades, 32(9), 32-39- recuperado de http://www.scielo.br/pdf/reeusp/v44n1/a05v44n1.pdf

Organización Mundial de la Salud, (2012). Recuperado el 13 de noviembre de 2013, de http://www.who.int/es/.

Organización Mundial de la Salud, publicación electrónica. Consultada el 9 de Junio de 2014 en la página: http:// www.who.int/mediacentre/factsheets/fs381/es/

Patiño, F. \& Cardona, D. (2007) Depresión en un grupo de jubilados participantes en programas de actividad física y/o asociaciones de la Universidad de Antioquia Medellín 2005. Revista Investigación y Educación en Enfermería, 25(1). Recuperado de http://redalyc.uaemex.mx/redalyc/pdf/1052/105215404009.pdf

Peña, D., Herazo, M. \& Calvo J. (2009). Depresión en ancianos. Revista Facultad de Medicina, 57(4),347- 355. Recuperado de http://www.scielo.org.co/scielo.php?script=sci_pdf\&pid=S012000112009000400007 \&lng=en\&nrm=iso\&tlng=es

Pérez, V. \& Arcia, N. (2007). Comportamiento de los factores biosociales en la depresión del adulto mayor. Recuperado de http://bvs.sld.cu/revistas/mgi/vol24_3_08/mgi02308.htm

Pérez, O. I. Ibáñez, P. M. Reyes, F. J. Atuesta, F. J. Suárez, D. M. (2008). Factores Asociados al Intento Suicida e Ideación Suicida Persistente en un Centro de Atención Primaria. Revista de Salud Pública, 10(3), 374-385. Recuperado de http://www.scielosp.org/pdf/rsap/v10n3/v10n3a02.pdf

Pandolfoa, Vázqueza, Mása, Vomeroa, Aguilara y Belloa, 2011. Prevalencia de la ideación e intento suicida en adolescentes de la Región Metropolitana de Santiago de Chile. Revista Médica de Chile, 138(3), 309 - 315. Recuperado de http://www.scielo.cl/scielo.php?script=sci_arttext\&pid=S0034-98872010000300008 
Piera, P., Pallero, R., Carrasco, C. \& Montorio, I (2010). Evaluación de la sintomatología depresiva en población mayor con pérdida visual: un estudio de la escala de depresión geriátrica. Psicothema, 22(4), 587-592. Recuperado de http://www.psicothema.com/pdf/3771.pdf

Ramón, F.; Guridi, M.; Medina, A. \& Dorta, Z. (2007). Comportamiento de la depresión en el adulto mayor. Psicología científica. Recuperado de http://www.psicologiacientifica.com/bv/psicologiapdf-301-comportamiento-de-la-depresion-en-el-adulto-mayor.pdf

Riquelme, M., Buendía, J. \& López, A. (2006). Desarrollo y validación de un instrumento para la evaluación de la depresión en ancianos. Psicothema, 18(2),288-292. Recuperado de http://www.psicothema.com/ pdf/3212.pdf

Salgado, N.; Gonzales, T.; Jáuregui, B. \& Bonilla, P. (2005). “No hacen viejos los años, sino los daños”: Envejecimiento y salud en varones rurales. Salud Pública de México, 47(4), 294-302. Recuperado de http:// www.scielo.br/pdf/spm/v47n4/a07v47n4.pdf

Sánchez, I., \& Castañeda, D. (2008). Prevalencia de depresión en mayores de 65 años. Perfil del anciano de riesgo. Centro de investigaciones sobre envejecimiento, longevidad y salud Manresa. Recuperado de http:// www.sld.cu/galerias/pdf/sitios/gericuba/articulo_depresion.pdf

Sánchez, Y., López, A., Calvo, M., Noriega, L. \& López, J. (2009). Depresión y deterioro cognitivo, estudio basado en la población de 65 años. Revista Habanera de Ciencias Médicas, 8(4). Recuperado de http://redalyc. uaemex.mx/src/inicio/ArtPdfRed.jsp?iCve=180414045008

Santos, T.; Silva, B \& Felicianni, V (2010). Diagnósticos e intervenciones de enfermería para ancianos con depresión y residentes en una institución de larga estancia (ILE). Revista Electrónica Cuatrimestral de Enfermería, 20, 1-13. Recuperado de http://scielo.isciii.es/pdf/eg/n20/clinica2.pdf

Tuesca, R., Fierro, N., Molinares, A., Oviedo, F., Polo, Y., Polo, J. \& Sierra, L., (2004). Los grupos de socialización como factor protector contra la depresión en personas ancianas. Revista Especializada de Salud Pública, 77, 595-604. Recuperado de http://scielo.isciii.es/pdf/resp/v77n5/original5.pdf

Uribe, A., Valderrama, L. \& Molina, L. (2010). Intervención psicológica en adultos mayores. Psicología desde el Caribe, 1(25), 246-258. Recuperado de http://www.redalyc.org/pdf/213/21315106011.pdf

\section{E 88}


Villanueva, M., (2005). Abordaje histórico de la psicología de la vejez. Revista de La Habana de Ciencias Médicas, 8(3). Recuperado de http://scielo.sld.cu/pdf/rhcm/v8n3/rhcm02309.pdf

Vinaccia, S., Gaviria, A. M., Atehortúa, L. F., Martínez, P. H., Trujillo, C. \& Quiceno, J. M. (2006). Prevalencia de depresión en niños escolarizados entre 8 y 12 años del oriente antioqueño a partir del "Child depression inventory"- CDI-.Diversitas-Perspectivas en Psicología, 2(1), 217-227. Recuperado de http:// www.scielo.unal.edu.co/scielo.php?script=sci_arttext\&pid=S1794-99982006000200004\&lng=es\&nr $\mathrm{m}=$ isso\&tlng=es

Vega J., Pereda B., Vega L. y Alonso A. (2003). Tendencia y factores de riesgo del suicidio en el adulto mayor. Recuperado de http://www.cocmed.sld.cu/no72/n72ori2.htm

Wagner, F. A., Gallo J. J. y Delva, J. (1999). Depression in late life: A hidden public health problem for Mexico? Revista de Salud Pública de México, 41(3), 189-202. Recuperado de http://www.redalyc.org/articulo. oa?id=10641307 


\section{Vejez, ¿un final "triste" para una historia de vida?. Revisión de estudios sobre la depresión en los adultos mayores}

Resumen | Desde finales del siglo pasado el mundo se enfrenta con el fenómeno del aumento de la esperanza de vida y por tanto del envejecimiento poblacional. El objetivo del presente artículo es mostrar una revisión a los estudios empíricos sobre el abordaje de la depresión durante la adultez mayor, tema que ha permanecido rezagado debido al conjunto de mitos, estereotipos y prejuicios que lo rodean. Se contemplaron 25 artículos, cuyos resultados se relacionan con la vulnerabilidad de los adultos mayores a sufrir de depresión, debido a los diversos cambios que atraviesan. Los artículos que se seleccionaron fueron artículos publicados desde el 2004 hasta el 2013, que se encontraron publicados en revistas indexadas en medio electrónico. Se encontró que algunos de los factores que influyen en la aparición de síntomas depresivos son: problemas de salud, jubilación, consumo de alcohol, dificultades en las relaciones interpersonales, disfunción familiar, limitaciones sensoriales, deterioro cognitivo, ausencia de vivienda y bajos ingresos económicos así como también estar vinculados a hogares de paso. En relación con el sexo, las investigaciones sugieren que los trastornos depresivos en la mujer son el doble de frecuentes que en los hombres. También se identificó que el medio ambiente cumple un papel importante en el control y prevención de los estados depresivos del adulto mayor, a su vez la integración familiar y social de las personas de edad avanzada permite disminuir el riesgo a desarrollar síntomas depresivos contribuyendo así al bienestar de los ancianos.

Palabras clave | Adultez mayor, depresión, factores de riesgo, suicidio.

\section{Citación}

Martínez-Baquero, L., Moreno-Heredia, C., \& Sánchez-Velasquez, X. (2014). Vejez, ¿Un final "triste" para una historia de vida?. Revisión de estudios sobre la depresión en los adultos mayores. Enfoques, 1(1). pp. 72-90.

Recibido: 09/06/2014 | Aceptado: 24/06/2014

\section{$\boldsymbol{E} \| 90$}

